1 Hacettepe Journal of Mathematics and Statistics

$\bigcap$ Volume 47 (3) (2018), 579-587

\title{
Ricci almost solitons on Concircular Ricci pseudosymmetric $\beta$-Kenmotsu manifolds
}

\author{
Shyamal Kumar Hui*† and Debabrata Chakraborty ${ }^{\ddagger}$
}

\begin{abstract}
The object of the present paper is to study concircular Ricci pseudosymmetric $\beta$-Kenmotsu manifolds whose metric is Ricci almost soliton. We found the conditions when Ricci almost soliton on concircular Ricci pseudosymmetric $\beta$-Kenmotsu manifold to be shrinking, steady and expanding respectively. We also construct an example of concircular Ricci pseudosymmetric $\beta$-Kenmotsu manifold whose metric is Ricci almost soliton.
\end{abstract}

Keywords: Ricci almost soliton, $\beta$-Kenmotsu manifold, Ricci pseudosymmetric manifold, concircular curvature tensor.

2000 AMS Classification: 53C15, 53C25

Received : 19.03.2017 Accepted : 20.04.2017 Doi : 10.15672/HJMS.2017.471

\section{Introduction}

In 1982, Hamilton [13] introduced the notion of Ricci flow to find a canonical metric on a smooth manifold. Then Ricci flow has become a powerful tool for the study of Riemannian manifolds, especially for those manifolds with positive curvature. Perelman ([24], [25]) used Ricci flow and its surgery to prove the Poincare conjecture. The Ricci flow is an evolution equation for metrics on a Riemannian manifold defined as follows:

$$
\frac{\partial}{\partial t} g_{i j}(t)=-2 R_{i j}
$$

A Ricci soliton emerges as the limit of the solutions of the Ricci flow. A solution to the Ricci flow is called Ricci soliton if it moves only by a one parameter group of

\footnotetext{
*Department of Mathematics, The University of Burdwan, Golapbag, Burdwan - 713104, West Bengal, India, Email: skhui@math.buruniv.ac. in

†Corresponding Author.

‡Department of Mathematics, Sidho Kanho Birsha University, Purulia - 723104, West Bengal, India, Email: debabratamath@gmail.com
} 
diffeomorphisms and scaling. To be precise a Ricci soliton on a Riemannian manifold $(M, g)$ is a triple $(g, V, \lambda)$ satisfying [14]

$$
£_{V} g+2 S+2 \lambda g=0,
$$

where $S$ is the Ricci tensor, $£_{V}$ is the Lie derivative along the vector field $V$ on $M$ and $\lambda \in \mathbb{R}$. The Ricci soliton is said to be shrinking, steady and expanding according as $\lambda$ is negative, zero and positive respectively.

During the last two decades, the geometry of Ricci solitons has been the focus of attention of many mathematicians. In particular, it has become more important after Perelman applied Ricci solitons to solve the long standing Poincare conjecture posed in 1904. In [30] Sharma studied the Ricci solitons in contact geometry. Thereafter Ricci solitons in contact metric manifolds have been studied by various authors such as Bagewadi et. al ([1], [2], [3], [19]), Bejan and Crasmareanu [4], Blaga [5], Calin and Crasmareanu [7], Chandra et. al [8], Chen and Deshmukh [9], Deshmukh et. al [10], Hui et. al ([15], [18]), Nagaraja and Premalatha [22], Tripathi [31] and many others.

In 1972, Kenmotsu [21] introduced a new class of almost contact Riemannian manifolds which are nowadays called Kenmotsu manifolds. It is well known that odd dimensional spheres admit Sasakian structures whereas odd dimensional hyperbolic spaces can not admit Sasakian structure, but have so-called Kenmotsu structure. Kenmotsu manifolds are normal (non-contact) almost contact Riemannian manifolds. Kenmotsu [21] investigated fundamental properties on local structure of such manifolds. Kenmotsu manifolds are locally isometric to warped product spaces with one dimensional base and Kähler fiber. As a generalization of both Sasakian and Kenmotsu manifolds, Oubiña [23] introduced the notion of trans-Sasakian manifolds, which are closely related to the locally conformal Kähler manifolds. A trans-Sasakian manifold of type $(0,0),(\alpha, 0)$ and $(0, \beta)$ are respectively called the cosympletic, $\alpha$-Sasakian and $\beta$-Kenmotsu manifold, $\alpha, \beta$ being scalar functions. In particular, if $\alpha=0, \beta=1$; and $\alpha=1, \beta=0$ then a transSasakian manifold will be a Kenmotsu and Sasakian manifold respectively. As $\beta$ is a scalar function, $\beta$-Kenmotsu manifolds provide a large varieties of Kenmotsu manifolds. $\beta$-Kenmotsu manifolds have been studied by several authors. In this connection it may be mentioned that Shaikh and Hui studied locally $\phi$-symmetric $\beta$-kenmotsu manifolds [28] and extended generalized $\phi$-recurrent $\beta$-Kenmotsu Manifolds [29], respectively. Also Calin and Crasmareanu [7] studied $f$-Kenmotsu manifolds.

The notion of Ricci pseudosymmetric manifold was introduced by Deszcz ([11],[12]). A geometrical interpretation of Ricci pseudosymmetric manifolds in the Riemannian case is given in [20]. A Riemannian manifold $\left(M^{n}, g\right)$ is called Ricci pseudosymmetric ([11], [12]) if the tensor $R \cdot S$ and the Tachibana tensor $Q(g, S)$ are linearly dependent, where

$$
\begin{aligned}
& (R(X, Y) \cdot S)(Z, U)=-S(R(X, Y) Z, U)-S(Z, R(X, Y) U), \\
& Q(g, S)(Z, U ; X, Y)=-S\left(\left(X \wedge_{g} Y\right) Z, U\right)-S\left(Z,\left(X \wedge_{g} Y\right) U\right),
\end{aligned}
$$

and

$$
\left(X \wedge_{g} Y\right) Z=g(Y, Z) X-g(X, Z) Y
$$

for all vector fields $X, Y, Z, U$ of $M, R$ denotes the curvature tensor of $M$. Then $\left(M^{n}, g\right)$ is Ricci pseudosymmetric if and only if

$$
(R(X, Y) \cdot S)(Z, U)=L_{S} Q(g, S)(Z, U ; X, Y)
$$

holds on $U_{S}=\left\{x \in M: S-\frac{r}{n} g \neq 0\right.$ at $\left.x\right\}$, for some function $L_{S}$ on $U_{S}$. If $R \cdot S=0$, then $M^{n}$ is called Ricci semisymmetric. Every Ricci semisymmetric manifold is Ricci pseudosymmetric but the converse is not true [12]. In this connection it is mentioned that Hui et. al ([17], [27]) studied Ricci pseudosymmetric generalized quasi-Einstein manifolds.

Recently Pigola et. al [26] introduced the notion of Ricci almost soliton and it can 
be defined on $\beta$-Kenmotsu manifold, if the relation (1.1) holds for smooth function $\lambda$. Thereafter Hui and Chakraborty [16] also studied Ricci almost solitons on generalized Sasakian-space-forms.

Motivated by the above studies the object of the present paper is to study concircular Ricci pseudosymmetric $\beta$-Kenmotsu manifold whose metric is a Ricci almost soliton. The paper is organized as follows. Section 2 is concerned with preliminaries. In section 3 , we investigate, Ricci almost solitons on concircular Ricci pseudosymmetric $\beta$-Kenmotsu manifolds and we found the value of $L_{S}$. Finally we construct an example of concircular Ricci pseudosymmetric $\beta$-Kenmotsu manifold whose metric is Ricci almost soliton through which the result of the paper is illustrated.

\section{Preliminaries}

A $(2 n+1)$-dimensional smooth manifold $M$ is said to be an almost contact metric manifold [6] if it admits an $(1,1)$ tensor field $\phi$, a vector field $\xi$, an 1-form $\eta$ and a Riemannian metric $g$, which satisfy

$$
\begin{aligned}
& \text { (a) } \phi \xi=0, \quad(b) \eta(\phi X)=0, \quad(c) \phi^{2} X=-X+\eta(X) \xi \\
& \begin{array}{l}
\text { (a) } g(\phi X, Y)=-g(X, \phi Y), \quad(b) \eta(X)=g(X, \xi), \quad(c) \eta(\xi)=1 \\
g(\phi X, \phi Y)=g(X, Y)-\eta(X) \eta(Y)
\end{array}
\end{aligned}
$$

for all $X, Y \in \chi(M)$. An almost contact metric manifold $M^{2 n+1}(\phi, \xi, \eta, g)$ is said to be a $\beta$-Kenmotsu manifold if the following conditions hold [21]:

$$
\begin{aligned}
& \nabla_{X} \xi=\beta[X-\eta(X) \xi], \\
& \left(\nabla_{X} \phi\right)(Y)=\beta[g(\phi X, Y) \xi-\eta(Y) \phi X] .
\end{aligned}
$$

If $\beta=1$, then a $\beta$-Kenmotsu manifold is called a Kenmotsu manifold; and if $\beta$ is constant, then it is called a homothetic Kenmotsu manifold. In a $\beta$-Kenmotsu manifold, the following relations hold ([21], [23]):

$$
\begin{aligned}
\left(\nabla_{X} \eta\right)(Y)=\beta[g(X, Y) & -\eta(X) \eta(Y)] \\
R(X, Y) \xi= & -\beta^{2}[\eta(Y) X-\eta(X) Y] \\
+ & (X \beta)\{Y-\eta(Y) \xi\}-(Y \beta)\{X-\eta(X) \xi\} \\
R(\xi, X) Y=\left[\beta^{2}+(\xi \beta)\right][ & \eta(Y) X-g(X, Y) \xi] \\
\eta(R(X, Y) Z)= & \beta^{2}[\eta(Y) g(X, Z)-\eta(X) g(Y, Z)] \\
& -(X \beta)\{g(Y, Z)-\eta(Y) \eta(Z)\} \\
& +(Y \beta)\{g(X, Z)-\eta(Z) \eta(X)\} \\
S(X, \xi)=-\left\{2 n \beta^{2}+(\xi \beta)\right\} & \eta(X)-(2 n-1)(X \beta)
\end{aligned}
$$

for all $X, Y, Z \in \chi(M)$.

Let $(g, \xi, \lambda)$ be a Ricci almost soliton on a $\beta$-Kenmotsu manifold $M^{2 n+1}(\phi, \xi, \eta, g)$. Then from (2.4), we get

$$
\begin{aligned}
\left(£_{\xi} g\right)(X, Y) & =g\left(\nabla_{X} \xi, Y\right)+g\left(X, \nabla_{Y} \xi\right) \\
& =\beta[g(X-\eta(X) \xi, Y)+g(X, Y-\eta(Y) \xi)] \\
& =2 \beta[g(X, Y)-\eta(X) \eta(Y)],
\end{aligned}
$$

i.e.

$$
\frac{1}{2}\left(£_{\xi} g\right)(X, Y)=\beta\{g(X, Y)-\eta(X) \eta(Y)\}
$$


From (1.1) and (2.11) we have

$$
S(X, Y)=-(\beta+\lambda) g(X, Y)+\beta \eta(X) \eta(Y),
$$

which yields

$$
\begin{aligned}
& Q X=-(\beta+\lambda) X+\beta \eta(X) \xi \\
& S(X, \xi)=-\lambda \eta(X) \\
& r=-(2 n+1) \lambda-2 n \beta
\end{aligned}
$$

where $Q$ is the Ricci operator, i.e., $g(Q X, Y)=S(X, Y)$ for all $X, Y$ and $r$ is the scalar curvature of $M^{2 n+1}(\phi, \xi, \eta, g)$.

\section{Concircular Ricci pseudosymmetric $\beta$-Kenmotsu manifolds and Ricci almost solitons}

This section deals with the study of Ricci almost solitons on concircular Ricci pseudosymmetric $\beta$-Kenmotsu manifolds. A concircular curvature tensor is an interesting invariant of a concircular transformation. A transformation of a $\beta$-Kenmotsu manifold $M^{2 n+1}(\phi, \xi, \eta, g)$, which transforms every geodesic circle of $M$ into a geodesic circle, is called a concircular transformation [32]. A concircular transformation is always a conformal transformation. Here geodesic circle means a curve in $M$ whose first curvature is constant and whose second curvature is identically zero. Thus the geometry of concircular transformations, that is, the concircular geometry, is a generalization of inversive geometry in the sense that the change of metric is more general than that induced by a circle preserving diffeomorphism. The interesting invariant of a concircular transformation is the concircular curvature tensor $\tilde{C}$, which is defined by [32]

$$
\tilde{C}(X, Y) Z=R(X, Y) Z-\frac{r}{2 n(2 n+1)}[g(Y, Z) X-g(X, Z) Y],
$$

where $R$ is the curvature tensor and $r$ is the scalar curvature of the $\beta$-Kenmotsu manifold $M^{2 n+1}(\phi, \xi, \eta, g)$.

Using (2.2), (2.7) and (2.9), we get

$$
\begin{aligned}
\tilde{C}(X, Y) \xi & =-\left[\beta^{2}+\frac{r}{2 n(2 n+1)}\right][\eta(Y) X-\eta(X) Y] \\
& +(X \beta)\{Y-\eta(Y) \xi\}-(Y \beta)\{X-\eta(X) \xi\}, \\
\eta(\tilde{C}(X, Y) U) & =\left[\beta^{2}+\frac{r}{2 n(2 n+1)}\right][\eta(Y) g(X, U)-\eta(X) g(Y, U)] \\
& -(X \beta)\{g(Y, U)-\eta(Y) \eta(U)\} \\
+ & (Y \beta)\{g(X, U)-\eta(X) \eta(U)\} .
\end{aligned}
$$

A $\beta$-Kenmotsu manifold $M^{2 n+1}(\phi, \xi, \eta, g), n>1$ is said to be concircular Ricci pseudosymmetric if its concircular curvature tensor $\tilde{C}$ satisfies

$$
(\tilde{C}(X, Y) \cdot S)(Z, U)=L_{S} Q(g, S)(Z, U ; X, Y)
$$

on $U_{S}=\left\{x \in M: S \neq \frac{r}{2 n+1} g\right.$ at $\left.x\right\}$, where $L_{S}$ is some function on $U_{S}$.

Let us take a concircular Ricci pseudosymmetric $\beta$-Kenmotsu manifold $M^{2 n+1}(\phi, \xi, \eta, g)$ whose metric is Ricci almost soliton. Then by virtue of (3.4) that

$$
\begin{array}{r}
S(\tilde{C}(X, Y) Z, U)+S(Z, \tilde{C}(X, Y) U)=L_{S}[g(Y, Z) S(X, U) \\
-g(X, Z) S(Y, U)+g(Y, U) S(X, Z)-g(X, U) S(Y, Z)] .
\end{array}
$$


By virtue of (2.12) it follows from (3.5) that

$$
\begin{aligned}
& \eta(\tilde{C}(X, Y) Z) \eta(U)+\eta(Z) \eta(\tilde{C}(X, Y) U) \\
= & L_{S}[g(Y, Z) \eta(X) \eta(U)-g(X, Z) \eta(Y) \eta(U) \\
+\quad & g(Y, U) \eta(X) \eta(Z)-g(X, U) \eta(Y) \eta(Z)] .
\end{aligned}
$$

Setting $Z=\xi$ in (3.6) and using (3.2) and (3.3), we get

$$
\begin{gathered}
{\left[L_{S}+\beta^{2}+\frac{r}{2 n(2 n+1)}\right][\eta(Y) g(X, U)-\eta(X) g(Y, U)]} \\
-\quad(X \beta)\{g(Y, U)-\eta(Y) \eta(U)\}+(Y \beta)\{g(X, U)-\eta(X) \eta(U)\}=0 .
\end{gathered}
$$

Putting $Y=\xi$ in (3.7) and using (2.2) and (2.15), we get

$$
\left.\left[L_{S}+\beta^{2}+(\xi \beta)-\frac{\lambda}{2 n}-\frac{\beta}{2 n+1}\right\}\right][g(X, U)+\eta(X) \eta(U)]=0
$$

for all vector fields $X$ and $U$, from which it follows that

$$
L_{S}=-\beta^{2}-(\xi \beta)+\frac{\lambda}{2 n}+\frac{\beta}{2 n+1} .
$$

This leads to the following :

3.1. Theorem. If $(g, \xi, \lambda)$ is a Ricci almost soliton on a concircular Ricci pseudosymmetric $\beta$-Kenmotsu manifold $M^{2 n+1}(\phi, \xi, \eta, g)$, then $L_{S}=-\beta^{2}-(\xi \beta)+\frac{\lambda}{2 n}+\frac{\beta}{2 n+1}$.

3.2. Corollary. In a concircular Ricci pseudosymmetric Kenmotsu manifold $M^{2 n+1}(\phi, \xi, \eta, g)$, the Ricci almost soliton $(g, \xi, \lambda)$ is shrinking, steady and expanding according as $L_{S}+\frac{2 n}{2 n+1}<0, L_{S}+\frac{2 n}{2 n+1}=0$ and $L_{S}+\frac{2 n}{2 n+1}>0$, respectively.

3.1. Example. We consider a 3-dimensional manifold $M=\left\{(x, y, z) \in \mathbb{R}^{3}: z \neq 0\right\}$, where $(x, y, z)$ are the standard coordinates in $\mathbb{R}^{3}$. Let $\left\{E_{1}, E_{2}, E_{3}\right\}$ be a linearly independent global frame on $M$ given by

$$
E_{1}=z^{2} \frac{\partial}{\partial x}, E_{2}=z^{2} \frac{\partial}{\partial y}, E_{3}=\frac{\partial}{\partial z}
$$

Let $g$ be the Riemannian metric defined by $g\left(E_{1}, E_{2}\right)=g\left(E_{1}, E_{3}\right)=g\left(E_{2}, E_{3}\right)=0$, $g\left(E_{1}, E_{1}\right)=g\left(E_{2}, E_{2}\right)=g\left(E_{3}, E_{3}\right)=1$. Let $\eta$ be the 1-form defined by $\eta(U)=g\left(U, E_{3}\right)$ for any $U \in \chi(M)$. Let $\phi$ be the $(1,1)$ tensor field defined by $\phi E_{1}=-E_{2}, \phi E_{2}=E_{1}$ and $\phi E_{3}=0$. Then using the linearity of $\phi$ and $g$ we have

$$
\eta\left(E_{3}\right)=1, \phi^{2} U=-U+\eta(U) E_{3}
$$

and

$$
g(\phi U, \phi W)=g(U, W)-\eta(U) \eta(W)
$$

for any $U, W \in \chi(M)$. Thus for $E_{3}=\xi,(\phi, \xi, \eta, g)$ defines an almost contact metric structure on $M$.

Let $\nabla$ be the Levi-Civita connection of $g$. Then we have

$$
\left[E_{1}, E_{2}\right]=0,\left[E_{1}, E_{3}\right]=-\frac{2}{z} E_{1},\left[E_{2}, E_{3}\right]=-\frac{2}{z} E_{2} .
$$

Using Koszul formula for the Riemannian metric $g$, we can easily calculate

$$
\begin{gathered}
\nabla_{E_{1}} E_{1}=\frac{2}{z} E_{3}, \nabla_{E_{1}} E_{2}=0, \nabla_{E_{1}} E_{3}=-\frac{2}{z} E_{1}, \\
\nabla_{E_{2}} E_{1}=0, \nabla_{E_{2}} E_{2}=\frac{2}{z} E_{3}, \nabla_{E_{2}} E_{3}=-\frac{2}{z} E_{2}, \\
\nabla_{E_{3}} E_{1}=0, \nabla_{E_{3}} E_{2}=0, \nabla_{E_{3}} E_{3}=0 .
\end{gathered}
$$

From the above it can be easily seen that for $E_{3}=\xi,(\phi, \xi, \eta, g)$ is a $\beta$-Kenmotsu structure on $M$. Consequently $M^{3}(\phi, \xi, \eta, g)$ is a $\beta$-Kenmotsu manifold with $\beta=-\frac{2}{z}$ [29]. 
Using the above relations, we can easily calculate the non-vanishing components of the curvature tensor as follows:

$$
\begin{aligned}
& R\left(E_{1}, E_{2}\right) E_{1}=\frac{4}{z^{2}} E_{2}, R\left(E_{1}, E_{2}\right) E_{2}=-\frac{4}{z^{2}} E_{1}, \\
& R\left(E_{1}, E_{3}\right) E_{1}=\frac{6}{z^{2}} E_{3}, R\left(E_{1}, E_{3}\right) E_{3}=-\frac{6}{z^{2}} E_{1}, \\
& R\left(E_{2}, E_{3}\right) E_{2}=\frac{6}{z^{2}} E_{3}, R\left(E_{2}, E_{3}\right) E_{3}=-\frac{6}{z^{2}} E_{2}
\end{aligned}
$$

and the components which can be obtained from these by the symmetry properties from which, we can easily calculate the non-vanishing components of the Ricci tensor as follows:

$$
S\left(E_{1}, E_{1}\right)=S\left(E_{2}, E_{2}\right)=-\frac{10}{z^{2}}, S\left(E_{3}, E_{3}\right)=-\frac{12}{z^{2}} .
$$

Also the scalar curvature $r$ is given by:

$$
r=-\frac{32}{z^{2}} \text {. }
$$

Since $\left\{E_{1}, E_{2}, E_{3}\right\}$ forms a basis of the 3-dimensional $\beta$-Kenmotsu manifold, any vector field $X, Y, Z, U \in \chi(M)$ can be written as

$$
\begin{aligned}
& X=a_{1} E_{1}+b_{1} E_{2}+c_{1} E_{3}, \\
& Y=a_{2} E_{1}+b_{2} E_{2}+c_{2} E_{3}, \\
& Z=a_{3} E_{1}+b_{3} E_{2}+c_{3} E_{3}, \\
& U=a_{4} E_{1}+b_{4} E_{2}+c_{4} E_{3},
\end{aligned}
$$

where $a_{i}, b_{i}, c_{i} \in \mathbb{R}^{+}$for all $i=1,2,3$ such that $a_{i}, b_{i}, c_{i}$ are not proportional. Then

$$
\begin{aligned}
R(X, Y) Z & =-\frac{2}{z^{2}}\left\{2 b_{3}\left(a_{1} b_{2}-a_{2} b_{1}\right)+3 c_{3}\left(a_{1} c_{2}-a_{2} c_{1}\right)\right\} E_{1} \\
& +\frac{2}{z^{2}}\left\{2 a_{3}\left(a_{1} b_{2}-a_{2} b_{1}\right)-3 c_{3}\left(b_{1} c_{2}-b_{2} c_{1}\right)\right\} E_{2} \\
& +\frac{6}{z^{2}}\left\{b_{3}\left(b_{1} c_{2}-b_{2} c_{1}\right)+a_{3}\left(a_{1} c_{2}-a_{2} c_{1}\right)\right\} E_{3}, \\
R(X, Y) U & =-\frac{2}{z^{2}}\left\{2 b_{4}\left(a_{1} b_{2}-a_{2} b_{1}\right)+3 c_{4}\left(a_{1} c_{2}-a_{2} c_{1}\right)\right\} E_{1} \\
& +\frac{2}{z^{2}}\left\{2 a_{4}\left(a_{1} b_{2}-a_{2} b_{1}\right)-3 c_{4}\left(b_{1} c_{2}-b_{2} c_{1}\right)\right\} E_{2} \\
& +\frac{6}{z^{2}}\left\{b_{4}\left(b_{1} c_{2}-b_{2} c_{1}\right)+a_{4}\left(a_{1} c_{2}-a_{2} c_{1}\right)\right\} E_{3} .
\end{aligned}
$$

In view of (3.10) we have from (3.1) that

$$
\begin{aligned}
\tilde{C}(X, Y) Z & =R(X, Y) Z-\frac{r}{6}[g(Y, Z) X-g(X, Z) Y] \\
& =-\frac{2}{z^{2}}\left[2 b_{3}\left(a_{1} b_{2}-a_{2} b_{1}\right)+3 c_{3}\left(a_{1} c_{2}-a_{2} c_{1}\right)\right. \\
& \left.-\frac{8}{3}\left\{a_{1}\left(b_{2} b_{3}+c_{2} c_{3}\right)-a_{2}\left(b_{1} b_{3}+c_{3} c_{1}\right)\right\}\right] E_{1} \\
& +\frac{2}{z^{2}}\left[2 a_{3}\left(a_{1} b_{2}-a_{2} b_{1}\right)-3 c_{3}\left(b_{1} c_{2}-b_{2} c_{1}\right)\right. \\
& \left.+\frac{8}{3}\left\{b_{1}\left(a_{2} a_{3}+c_{2} c_{3}\right)-b_{2}\left(a_{1} a_{3}+c_{3} c_{1}\right)\right\}\right] E_{2} \\
& +\frac{2}{z^{2}}\left[3\left\{b_{3}\left(b_{1} c_{2}-b_{2} c_{1}\right)+a_{3}\left(a_{1} c_{2}-a_{2} c_{1}\right)\right\}\right. \\
& \left.+\frac{8}{3}\left\{c_{1}\left(a_{2} a_{3}+b_{2} b_{3}\right)-c_{2}\left(a_{1} a_{3}+b_{1} b_{3}\right)\right\}\right] E_{3} .
\end{aligned}
$$


Hence

$$
\begin{aligned}
& S(\tilde{C}(X, Y) Z, U) \\
= & \frac{20 a_{4}}{z^{4}}\left[2 b_{3}\left(a_{1} b_{2}-a_{2} b_{1}\right)+3 c_{3}\left(a_{1} c_{2}-a_{2} c_{1}\right)\right. \\
- & \left.\frac{8}{3}\left\{a_{1}\left(b_{2} b_{3}+c_{2} c_{3}\right)-a_{2}\left(b_{1} b_{3}+c_{3} c_{1}\right)\right\}\right] \\
- & \frac{20 b_{4}}{z^{4}}\left[2 a_{3}\left(a_{1} b_{2}-a_{2} b_{1}\right)-3 c_{3}\left(b_{1} c_{2}-b_{2} c_{1}\right)\right. \\
+ & \left.\frac{8}{3}\left\{b_{1}\left(a_{2} a_{3}+c_{2} c_{3}\right)-b_{2}\left(a_{1} a_{3}+c_{3} c_{1}\right)\right\}\right] \\
- & \frac{24 c_{4}}{z^{4}}\left[3\left\{b_{3}\left(b_{1} c_{2}-b_{2} c_{1}\right)+a_{3}\left(a_{1} c_{2}-a_{2} c_{1}\right)\right\}\right. \\
+ & \left.\frac{8}{3}\left\{c_{1}\left(a_{2} a_{3}+b_{2} b_{3}\right)-c_{2}\left(a_{1} a_{3}+b_{1} b_{3}\right)\right\}\right] .
\end{aligned}
$$

Similarly we obtain

$$
\begin{aligned}
& S(Z, \tilde{C}(X, Y) U) \\
= & \frac{20 a_{3}}{z^{4}}\left[2 b_{4}\left(a_{1} b_{2}-a_{2} b_{1}\right)+3 c_{4}\left(a_{1} c_{2}-a_{2} c_{1}\right)\right. \\
- & \left.\frac{8}{3}\left\{a_{1}\left(b_{2} b_{4}+c_{2} c_{4}\right)-a_{2}\left(b_{1} b_{4}-c_{1} c_{4}\right)\right\}\right] \\
- & \frac{20 b_{3}}{z^{4}}\left[2 a_{4}\left(a_{1} b_{2}-a_{2} b_{1}\right)-3 c_{4}\left(b_{1} c_{2}-b_{2} c_{1}\right)\right. \\
+ & \left.\frac{8}{3}\left\{b_{1}\left(a_{2} a_{4}+c_{2} c_{4}\right)-b_{2}\left(a_{1} a_{4}+c_{1} c_{4}\right)\right\}\right] \\
- & \frac{24 c_{3}}{z^{4}}\left[3\left\{b_{4}\left(b_{1} c_{2}-b_{2} c_{1}\right)+a_{4}\left(a_{1} c_{2}-a_{2} c_{1}\right)\right\}\right. \\
+ & \left.\frac{8}{3}\left\{c_{1}\left(a_{2} a_{4}+b_{2} b_{4}\right)-c_{2}\left(a_{1} a_{4}+b_{1} b_{4}\right)\right\}\right] .
\end{aligned}
$$

Now we have

$$
\left\{\begin{array}{l}
g(Y, Z)=a_{2} a_{3}+b_{2} b_{3}+c_{2} c_{3} \\
g(X, Z)=a_{1} a_{3}+b_{1} b_{3}+c_{1} c_{3} \\
g(Y, U)=a_{2} a_{4}+b_{2} b_{4}+c_{2} c_{4} \\
g(X, U)=a_{1} a_{4}+b_{1} b_{4}+c_{1} c_{4} .
\end{array}\right.
$$

Also we have

$$
\left\{\begin{array}{l}
S(Y, Z)=-\frac{2}{z^{2}}\left(5 a_{2} a_{3}+5 b_{2} b_{3}+6 c_{2} c_{3}\right), \\
S(X, Z)=-\frac{2}{z^{2}}\left(5 a_{1} a_{3}+5 b_{1} b_{3}+6 c_{1} c_{3}\right), \\
S(Y, U)=-\frac{2}{z^{2}}\left(5 a_{2} a_{4}+5 b_{2} b_{4}+6 c_{2} c_{4}\right), \\
S(X, U)=-\frac{2}{z^{2}}\left(5 a_{1} a_{4}+5 b_{1} b_{4}+6 c_{1} c_{4}\right) .
\end{array}\right.
$$

Therefore from (3.14) and (3.15) we have

$$
\begin{aligned}
& g(Y, Z) S(X, U)-g(X, Z) S(Y, U) \\
+ & g(Y, U) S(X, Z)-g(X, U) S(Y, Z) \\
= & \frac{2}{z^{2}}\left[\left(a_{1} c_{2}-a_{2} c_{1}\right)\left(a_{3} c_{4}+a_{4} c_{3}\right)+\left(b_{1} c_{2}-b_{2} c_{1}\right)\left(b_{3} c_{4}+b_{4} c_{3}\right)\right] \\
\neq & 0,
\end{aligned}
$$

since $a_{i}, b_{i}, c_{i}$ are not proportional and assume that $\left(a_{1} c_{2}-a_{2} c_{1}\right)\left(a_{3} c_{4}+a_{4} c_{3}\right)+\left(b_{1} c_{2}-\right.$ $\left.b_{2} c_{1}\right)\left(b_{3} c_{4}+b_{4} c_{3}\right) \neq 0$. 
Also from (3.12) and (3.13) we get

$$
\begin{aligned}
& S(\tilde{C}(X, Y) Z, U)+S(Z, \tilde{C}(X, Y) U) \\
= & \frac{44}{3 z^{4}}\left[\left(a_{1} c_{2}-a_{2} c_{1}\right)\left(a_{3} c_{4}+a_{4} c_{3}\right)+\left(b_{1} c_{2}-b_{2} c_{1}\right)\left(b_{3} c_{4}+b_{4} c_{3}\right)\right] \\
\neq & 0 .
\end{aligned}
$$

Let us consider the function

$$
\text { (3.18) } \quad L_{S}=\frac{22}{3 z^{2}} \text {. }
$$

By virtue of (3.18) we have from (3.16) and (3.17) that

$$
\begin{aligned}
& S(\tilde{C}(X, Y) Z, U)+S(Z, \tilde{C}(X, Y) U)=L_{S}[g(Y, Z) S(X, U) \\
-\quad & g(X, Z) S(Y, U)+g(Y, U) S(X, Z)-g(X, U) S(Y, Z)] .
\end{aligned}
$$

Hence the $\beta$-Kenmotsu manifold $M^{3}(\phi, \xi, \eta, g)$ is concircular Ricci pseudosymmetric. If $(g, \xi, \lambda)$ is a Ricci almost soliton on this $\beta$-Kenmotsu manifold $M^{3}(\phi, \xi, \eta, g)$, then from (2.15) we get

$$
r=-3 \lambda-2 \beta
$$

i.e.,

i.e.,

$$
-\frac{32}{z^{2}}=-3 \lambda+\frac{4}{z}
$$

and hence from (3.9) we get

$$
\lambda=\frac{4}{3}\left(\frac{1}{z}+\frac{8}{z^{2}}\right)
$$

$$
\begin{aligned}
L_{S} & =-\beta^{2}-(\xi \beta)+\frac{\lambda}{2}+\frac{\beta}{3} \\
& =\frac{22}{3 z^{2}}, \quad \text { as } \beta=-\frac{2}{z}, \xi=E_{3}=\frac{\partial}{\partial z},
\end{aligned}
$$

which satisfies (3.18). Thus Theorem (3.1) is verified.

Acknowledgments. The authors would like to thank the referees for their comments that helped us to improve this article.

\section{References}

[1] Ashoka, S. R., Bagewadi, C. S. and Ingalahalli, G., Certain results on Ricci solitons in $\alpha$ Sasakian manifolds, Hindawi Publ. Corporation, Geometry, Vol. 2013, Article ID 573925, 4 pages.

[2] Ashoka, S. R., Bagewadi, C. S. and Ingalahalli, G., A geometry on Ricci solitons in $(L C S)_{n}$ manifolds, Diff. Geom.-Dynamical Systems, 16 (2014), 50-62.

[3] Bagewadi, C. S. and Ingalahalli, G., Ricci solitons in Lorentzian $\alpha$-Sasakian manifolds, Acta Math. Acad. Paeda. Nyire., 28 (2012), 59-68.

[4] Bejan, C. L. and Crasmareanu, M., Ricci solitons in manifolds with quasi constant curvature, Publ. Math. Debrecen, 78 (2011), 235-243.

[5] Blaga, A. M., $\eta$-Ricci solitons on para-kenmotsu manifolds, Balkan J. Geom. Appl., 20 (2015), 1-13.

[6] Blair, D. E., Contact manifolds in Riemannian geometry, Lecture Notes in Math. 509, Springer-Verlag, 1976.

[7] Calin, C. and Crasmareanu, M., From the Eisenhart problem to Ricci solitons in $f$ Kenmotsu manifolds, Bull. Malays. Math. Sci. Soc. (2), 33 (2010), 361-368.

[8] Chandra, S., Hui, S. K. and Shaikh, A. A., Second order parallel tensors and Ricci solitons on $(L C S)_{n}$-manifolds, Commun. Korean Math. Soc., 30 (2015), 123-130.

[9] Chen, B. Y. and Deshmukh, S., Geometry of compact shrinking Ricci solitons, Balkan J. Geom. Appl., 19 (2014), 13-21. 
[10] Deshmukh, S., Al-Sodais, H. and Alodan, H., A note on Ricci solitons, Balkan J. Geom. Appl.,16 (2011), 48-55.

[11] Deszcz, R., On Ricci-pseudosymmetric warped products, Demonstratio Math., 22 (1989), $1053-1065$.

[12] Deszcz R., On pseudosymmetric spaces, Bull. Soc. Math. Belg. Sér. A, 44(1) (1992), 1-34.

[13] Hamilton, R. S., Three-manifolds with positive Ricci curvature, J. Diff. Geom., 17 (1982), $255-306$.

[14] Hamilton, R. S., The Ricci flow on surfaces, Mathematics and general relativity, Contemp. Math., 71, American Math. Soc., 1988, 237-262.

[15] Hui, S. K. and Chakraborty, D., Some types of Ricci solitons on $(L C S)_{n}$-manifolds, J. Math. Sci. Advances and Applications, 37 (2016), 1-17.

[16] Hui, S. K. and Chakraborty, D., Generalized Sasakian-space-forms and Ricci almost solitons with a conformal killing vector field, New Trends in Math. Sci., 4 (2016), 263-269.

[17] Hui, S. K. and Lemence, R. S., Ricci pseudosymmetric generalized quasi-Einstein manifolds, SUT J. Math., 51 (2015), 67-85.

[18] Hui, S. K., Lemence, R. S. and Chakraborty, D., Ricci solitons on three dimensional generalized Sasakian-space-forms, Tensor Society, N. S., 76 (2015), 75-83.

[19] Ingalahalli, G. and Bagewadi, C. S., Ricci solitons in $\alpha$-Sasakian manifolds,Hindawi Publishing Corporation, ISRN Geometry, Vol. 2012, Article ID 421384, 13 pages.

[20] Jahanara, B., Haesen, S., Sentürk, Z. and Verstraelen, L., On the parallel transport of the Ricci curvatures, J. Geom. Phys., 57 (2007), 1771-1777.

[21] Kenmotsu, K., A class of almost contact Riemannian manifolds, Tohoku Math. J., 24 (1972), 93-103.

[22] Nagaraja, H. G. and Premalatha, C. R., Ricci solitons in Kenmotsu manifolds, J. Math. Analysis, 3 (2012), 18-24.

[23] Oubiña, J. A., New classes of almost contact metric structures, Publ. Math. Debrecen, 32 (1985), 187-193.

[24] Perelman, G., The entropy formula for the Ricci flow and its geometric applications, http://arXiv.org/abs/math/0211159, 2002, 1-39.

[25] Perelman, G., Ricci flow with surgery on three manifolds, http://arXiv.org/abs/math/0303109, 2003, 1-22.

[26] Pigola, S., Rigoli, M., Rimoldi, M. and Setti, A. G., Ricci almost solitons, Ann. Sc. Norm. Super. Pisa Cl. Sci, 10 (2011), 757-799.

[27] Shaikh, A. A. and Hui, S. K., On some classes of generalized quasi-Einstein manifolds, Commun. Korean Math. Soc., 24(3) (2009), 415-424.

[28] Shaikh, A. A. and Hui, S. K., On locally $\phi$-symmetric $\beta$-kenmotsu manifolds, Extracta Mathematicae, 24(3) (2009), 301-316.

[29] Shaikh, A. A. and Hui, S. K., On extended generalized $\phi$-recurrent $\beta$-Kenmotsu Manifolds, Publ. de l'Institut Math. (Beograd), 89(103) (2011), 77-88.

[30] Sharma, R., Certain results on $k$-contact and $(k, \mu)$-contact manifolds, J. of Geom., 89 (2008), 138-147.

[31] Tripathi, M. M., Ricci solitons in contact metric manifolds, arxiv:0801.4221 [Math.DG] (2008).

[32] Yano, K., Concircular geometry, I, Proc. Imp. Acad. Tokyo, 16 (1940), 195-200. 